\title{
Pulsations of Long-Period Variables and their Connection to Variations of Circumstellar Maser Emission
}

\author{
G. M. Rudnitskij \\ Sternberg Astronomical Institute, Moscow State University, Moscow, \\ 119899, Russia,gmr@sai.msk.su
}

\begin{abstract}
I consider maser emission in the $\mathrm{H}_{2} \mathrm{O}$ spectral line $\lambda=$ $1.35 \mathrm{~cm}$ from long-period variable stars. Some models for $\mathrm{H}_{2} \mathrm{O}$ maser variability are suggested.
\end{abstract}

Many of long-period variables (LPVs - Miras and semiregulars) emit in maser spectral lines of molecules at microwaves. I consider in more detail $\mathrm{H}_{2} \mathrm{O}$ masers, common in variable oxygen-rich red giants.

I use data of LPV monitoring in the $\mathrm{H}_{2} \mathrm{O}$ line, obtained on the 22-meter radio telescope in Pushchino jointly with our colleagues from the Lebedev Physical Institute, Russian Academy of Sciences. For earlier results see Berulis et al. (1983). Literature data are used, too. The curves of the $\mathrm{H}_{2} \mathrm{O}$ line flux variability $F(t)$ were compared to optical light curves of the stars (Berulis et al. 1984, Rudnitskij 1987, Kudashkina \& Rudnitskij 1988). In many cases (R Aql, $\mathrm{R}$ Leo, U Ori, U Her), there is a good correlation of the $\mathrm{H}_{2} \mathrm{O}$ line flux density with the optical brightness. There is some delay of the $F(t)$ curves with respect to the optical curves, by about $0.1-0.3 P$ ( $P$ is the period of light variations). I consider the following mechanisms that may account for such a correlation.

a. Direct impact of a shock on the maser generation region (Rudnitskij \& Chuprikov 1990). The mechanism involves nonequilibrium conditions just behind the shock front (with gas particles heated immediately, while dust grains remain cooler for some time). Thus, a nonequilibrium post-shock gas layer is formed; conditions in it are favourable for the maser.

b. Amplification of the free-free radio continuum emitted by postshock ionized gas (Rudnitskij 1990). In this mechanism, $\mathrm{H}_{2} \mathrm{O}$ molecules amplify the radio continuum at $\lambda=1.35 \mathrm{~cm}$ from the ionized post-shock gas. This gas is optically thick down to wavelengths as short as $1 \mathrm{~cm}$. Thus, its brightness temperature, $T_{b}=(15-50) \times 10^{3} \mathrm{~K}$, is high enough to stimulate the $\mathrm{H}_{2} \mathrm{O}$ maser, especially at visual phases $\varphi=0.1-0.3$, in accordance with the above-mentioned correlation between the $\mathrm{H}_{2} \mathrm{O}$ maser intensity and the optical light curve.

c. Amplification of the nonthermal radio continuum. The effect itself is analogous to the previous one. It may cause strong, short-lived bursts of the $\mathrm{H}_{2} \mathrm{O}$ maser, which would repeat the time shape of the radio continuum bursts. This is a more hypothetical possibility, although strong nonthermal radio continuum was observed, at least twice, in R Aql (Woodsworth \& Hughes 1973, Estalella et al. 1983).

In many stars, the $\mathrm{H}_{2} \mathrm{O}$ line profiles are simple during most time, consisting of a single emission peak at roughly the stellar radial velocity $V_{*}$. But, almost in every star studied (e.g., R Aql, U Ori, U Lyn, W Hya, etc.), there were 
episodes when the $\mathrm{H}_{2} \mathrm{O}$ line profile became more complicated, with multiple features. Most of these features were blue-shifted with respect to $V_{*}$, with emission extending sometimes at $10 \mathrm{~km} / \mathrm{s}$ blueward. Some of the features exceeded the main peak in flux density. The effect is most prominent in LPVs classified SR (RT Vir, RX Boo, W Hya). From a comparison with optical light curves, we found that such episodes might follow the brighter visual maxima.

We present a model, in which the $\mathrm{H}_{2} \mathrm{O}$ maser is excited by a shock wave, propagating with a velocity $D_{0} \sim 6-10 \mathrm{~km} / \mathrm{s}$ in the inner part of the circumstellar envelope during each cycle of light variability. The profile of the $\mathrm{H}_{2} \mathrm{O}$ line and the maser strength depend on the shock velocity in a given light cycle. There are indications that the episodic appearance of blue-shifted maser features is more frequent in stars with bumps on the ascending branch of their light curves (e.g, U Her, U Lyn). In our previous work (Kudashkina \& Rudnitskij 1995), we connected the bumps and their duration with propagation of the shock wave in the stellar atmosphere.

Another item is the asymmetry in spatial distribution of circumstellar maser emission, implying lack of spherical symmetry of the mass loss process (bipolar maser distribution in R Aql, U Ori). This also yields characteristic structures in the $\mathrm{H}_{2} \mathrm{O}$ line profiles (triple profile or pairs of double features). We connect this with probable nonradial pulsations of the star, resulting in an equatorial torus of circumstellar matter, while masers flow out mainly in the polar directions.

Acknowledgments. This work was supported by the Russian Foundation for Fundamental Research (Project Code 94-02-05763). The author gratefully acknowledges also the financial assistance from the International Science Foundation (Travel Grant No. 4546/4).

\section{References}

Berulis, I. I., Lekht, E. E., Pashchenko, M. I., \& Rudnitskii G. M. 1983, Soviet Ast., 27, 179

Berulis, I. I., et al. 1984, Nauchnye Informatsii, Astron. Council, Acad. Sci. USSR, 56, 92

Estalella, R., Paredes, J. M., \& Ruis, A. 1983, A\&A, 124, 309

Kudashkina, L. S., \& Rudnitskij, G. M. 1988, Peremennye Zvezdy, 22, 925

Kudashkina, L. S., \& Rudnitskij, G. M. 1995, Odessa Astron. Publ., Vol.7 (in press)

Rudnitskij G. M. 1987, in Circumstellar Matter, Proceedings of the 122nd Symposium of the International Astronomical Union, Held in Heidelberg, F. R. Germany 23-27 June 1986, I .Appenzeller \& C. Jordan, Dordrecht: Reidel, 267

Rudnitskij, G. M. 1990, in Proceedings of the International Colloquium From Miras to Planetary Nebulae: Which Path for Stellar Evolution? Montpellier, France, September 4-7, 1989, M. O. Mennessier \& A. Omont, Gif sur Yvette: Editions Frontières, 268

Rudnitskii, G. M., \& Chuprikov, A. A. 1990, Soviet Ast., 34, 147

Woodsworth, A. W., \& Hughes, V. A. 1973, Nature, Phys. Sci., 246, 111 